DOI: $10.3901 / J M E .2020 .22 .190$

\title{
考虑摩擦副接触应力场和冷却流场的湿式 离合器温度场分析
}

\author{
吴邦治 ${ }^{1}$ 秦大同 ${ }^{1,2}$ 胡建军 ${ }^{1,2}$ 刘永刚 ${ }^{1,2}$ \\ (1. 重庆大学机械传动国家重点实验室 重庆 400044; \\ 2. 重庆大学汽车工程学院 重庆 400044)
}

\begin{abstract}
摘要: 温度对湿式离合器摩擦副的摩擦特性和热失效具有重要影响。为了获取湿式离合器温度场的分布规律, 建立摩擦副接 触应力分布有限元模型和摩擦片沟槽内冷却流场数值计算模型, 获得了摩擦副接触应力随离合器接合油压的变化规律和冷却 流场对流换热随离合器转速的变化规律。在此基础上, 提出考虑离合器摩擦副接触应力分布时变特性和冷却流场分布时变特 性的离合器温度场数值计算模型。将所建温度场模型的仿真结果与试验结果作对比, 验证了所建温度场模型的正确性。通过 计算获得了湿式离合器接合过程中不同钢片在半径和厚度方向的温度分布规律，揭示了摩擦副接触应力场和摩擦片沟槽内冷 却流场对离合器温度场的影响规律。结果表明, 在离合器摩擦副半径方向上, 摩擦副的温度分布规律与接触应力分布规律相 一致。而摩擦片沟槽内冷却流场的对流换热主要影响离合器同步阶段的温度分布。
\end{abstract}

关键词: 湿式离合器; 应力场; 冷却流场; 温度场; 数值计算

中图分类号: U463

\section{Analysis of Temperature Field of Wet Clutch Considering Contact Stress Field and Cooling Flow Field of Friction Pair}

\author{
WU Bangzhi ${ }^{1}$ QIN Datong ${ }^{1,2}$ HU Jianjun ${ }^{1,2}$ LIU Yonggang ${ }^{1,2}$ \\ (1. State Key Laboratory of Mechanical Transmission, Chongqing University, Chongqing 400044; \\ 2. College of Automotive Engineering, Chongqing University, Chongqing 400044)
}

\begin{abstract}
Temperature plays an important role in friction characteristics and thermal failure of wet clutch friction pairs. In order to obtain the distribution of the temperature field of the wet clutch, the finite element model of the contact stress distribution of the friction pair and the numerical calculation model of the cooling flow field in the groove of the friction plate are established. The variation of the contact stress of the friction pair with the clutch engagement oil pressure and the variation of convective heat transfer in the cooling flow field with the clutch speed are obtained. On this basis, a numerical model of clutch temperature field considering the time-varying characteristics of contact stress distribution and cooling flow field distribution is proposed. The simulation results of the temperature field model are compared with the experimental results to verify the correctness of the temperature field model. The temperature distribution of different separator plates in the radius and thickness directions during the wet clutch engagement process is obtained. The influences of the contact stress field of the friction pair and the cooling flow field in the groove of the friction plate on the temperature field of the clutch are revealed. The results show that the temperature distribution is consistent with the contact stress distribution in the radial direction of the clutch friction pair. The convective heat transfer of the cooling flow field in the groove of the friction plate mainly affects the temperature distribution of the clutch locked phase.
\end{abstract}

Key words: wet clutch; stress field; flow field; temperature field; numerical calculation

\section{0 前言}

湿式离合器被广泛应用于车辆动力传动, 如双

* 国家自然科学基金(U1764259)、中央高校基础研究(2018CDJDCD0002) 和重庆基础研究与前沿探索(cstc2018jcyj AX0409)资助项目。20200122 收到初稿, 20200926 收到修改稿
离合自动变速器。但湿式离合器在接合过程中, 由 于滑摩会产生大量的摩擦热。摩擦热会改变摩擦副 的摩擦磨损等特性, 甚至会引起摩擦副的热失效。 在车辆的起步和换挡过程中, 离合器摩擦副的摩擦 特性变化会引起离合器传递转矩的波动, 造成车辆 的纵向冲击, 影响车辆的驾驶舒适性 ${ }^{[1]}$ 。另外, 车 
辆在恶劣的行驶工况下, 离合器的频繁接合会产生 大量的摩擦热, 从而造成离合器温升过高。过高的 离合器温度将会造成离合器摩擦副的热失效, 影响 车辆的驾驶安全性。

湿式离合器温度场主要由冷却流场的对流换 热和摩擦副的热流密度确定, 其中摩擦副的热流 密度由摩擦副转速差和摩擦副接触应力计算得 到。目前关于离合器温度场的研究, 文献[2]采用 解析法求解了湿式离合器的温度分布, 并对湿式 离合器温度分布进行了试验研究。文献[3]采用解 析法求解了制动器的三维温度分布, 分析了制动 器温度场的分布规律。解析法虽然能够获得温度 分布的准确解, 但对于考虑摩擦副接触应力场和冷 却流场时变特性的温度场模型无法求解。文献[4] 建立了湿式多片离合器等效热平衡方程, 分析了 离合器初始温度、摩擦片质量以及冷却流量对湿 式离合器温度变化的影响。虽然等效热平衡方程 的求解方便, 但无法获取离合器温度场在半径和 厚度方向的分布规律。文献[5-8]建立了离合器温 度场数值模型, 分析了离合器接合过程中温度场 的变化规律及影响因素。文献[9]利用有限元法仿 真分析了不同初始制动速度对制动器三维温度场 的影响。数值方法虽能用于求解考虑摩擦副接触 应力场和冷却流场时变特性的温度场模型, 但上 述的温度场数值模型均假设摩擦副的接触应力均 匀分布和冷却流场的对流换热系数恒定不变。

在考虑摩擦副接触应力场对离合器温度场的影 响方面, 文献[10]建立了湿式离合器摩擦副接触应 力场和温度场有限元模型, 分析了摩擦副在集中力 作用下的接触应力场对温度场的影响规律。文献 [11-15]建立了摩擦副温度场和应力场耦合数值模 型, 揭示了摩擦副温度场与热应力之间的耦合机理。 文献[16]建立了湿式离合器温度场和应力场有限元 模型, 分析了摩擦片厚度、比热容以及导热系数对 离合器温度场和应力场的影响规律。但对于离合器 接合过程, 上述研究并未考虑摩擦接触应力随离合 器接合油压变化的时变特性。

在考虑冷却流场对流换热对离合器温度场的 影响方面, 文献[17]建立了湿式离合器温度场有 限元模型, 仿真分析了沟槽内冷却流场对离合器 温度场分布的影响。文献[18]基于对摩擦片沟槽 内冷却流场对流换热系数的试验拟合, 建立了湿 式离合器接合过程中的温度场数值模型。文献[19] 采用有限体积法求解了湿式离合器温度场模型, 仿真分析了不同离合器沟槽内冷却流场的散热性
能。文献[20]建立了湿式离合器温度场等效热平 衡模型, 分析了沟槽类型、冷却流量以及转速对 离合器温度场的影响。但对于离合器接合过程, 上述研究并未考虑冷却流场的对流换热随离合器 转速变化的时变特性。

综上所述, 目前缺乏综合考虑摩擦副接触应 力场和冷却流场的湿式离合器温度场数值计算模 型。并且对于离合器的接合过程, 摩擦副的接触 应力场和冷却流场将随着离合器接合油压和转速 的变化而变化。然而对于湿式离合器接合过程的 温度场分析, 已有研究并未考虑摩擦副的接触应 力场和冷却流场随着离合器接合油压和转速变化 的时变特性。

\section{1 离合器摩擦副接触应力场分析}

对于离合器的接合过程, 摩擦副的接触应力随 着离合器接合油压的变化而变化。通过对摩擦副的 受力分析, 建立了摩擦副接触应力分布有限元模型, 从而获取了摩擦副接触应力随接合油压变化的分布 规律。

图 1 为湿式离合器结构组成。液压缸在接合油 压的推动下, 克服膜片弹簧的阻力压紧摩擦副。摩 擦副在液压缸的压紧力和卡簧的固定约束作用下传 递扭矩, 同时产生摩擦热。液压缸的压紧力由接合 油压和膜片弹簧的阻力确定。接合油压为离合器接 合过程的可控值。而膜片弹簧的阻力由膜片的变形 量和膜片的刚度计算得到。

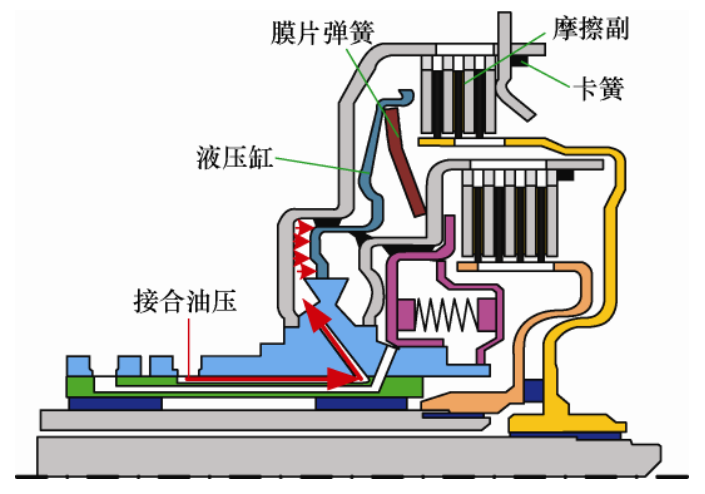

图 1 湿式离合器结构组成

\section{1 膜片弹簧刚度分析}

在离合器接合过程中, 液压缸和摩擦副的间距 即为膜片弹簧的变形量。而膜片弹簧的刚度随着膜 片变形量的变化而变化。膜片弹簧刚度可由力与变 形的关系确定。 
图 2 所示为膜片弹簧力与变形的关系。图中显 示了多组试验的最终结果, 给出了最大值、最小值 和标称值。

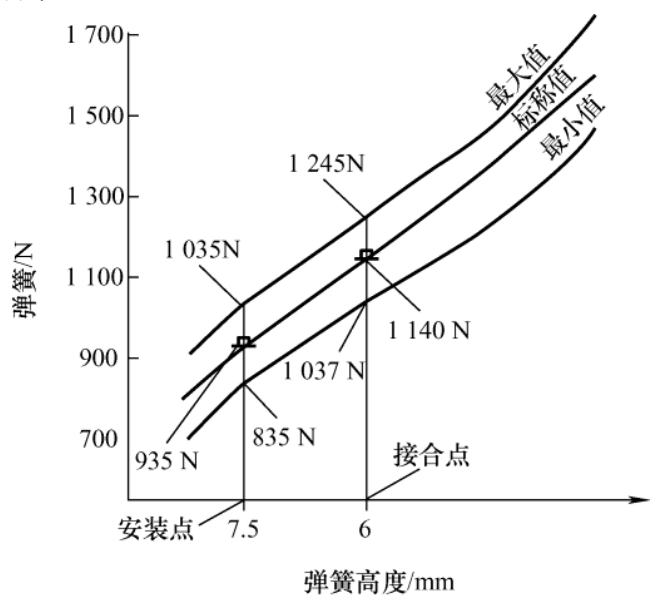

图 2 膜片弹簧力与变形曲线

\section{2 摩擦副受力分析}

图 3 为摩擦副轴向受力分析。液压缸在接合油 压的作用下克服膜片弹簧的阻力压紧摩擦副, 同时 摩擦副受轴向固定约束。

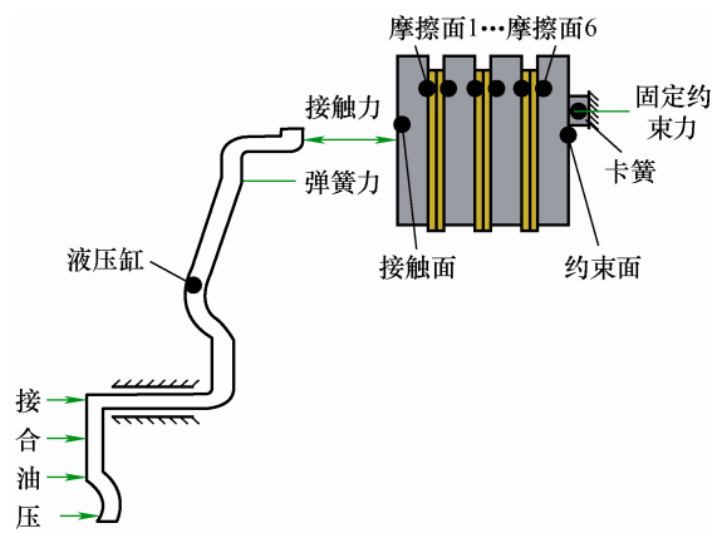

图 3 摩擦副轴向受力分析

\section{3 摩擦副接触应力分布}

图 4a 为接合油压在 $1 \mathrm{MPa}$ 时, 不同摩擦副的 接触应力在半径方向上的分布规律。由于液压缸的 压紧力和固定端的约束力为集中力, 且集中力位于 摩擦副径向的中部。因此摩擦副接触应力在径向分 布为中间大两端小, 呈抛物线分布。本文离合器的 摩擦片材料为纸基材料, 相对柔软。因此集中力经 过纸基摩擦片时, 中间摩擦副的接触应力分布不均 程度被削弱。

图 $4 \mathrm{~b}$ 为摩擦面平均半径处接触应力随油压的 变化规律。随着接合油压的增加, 摩擦副接触应力 呈线性增加。且中间四个摩擦面的接触应力变化率 基本相同。但第一和第六摩擦面的接触应力变化率 大于中间摩擦面的接触应力变化率。

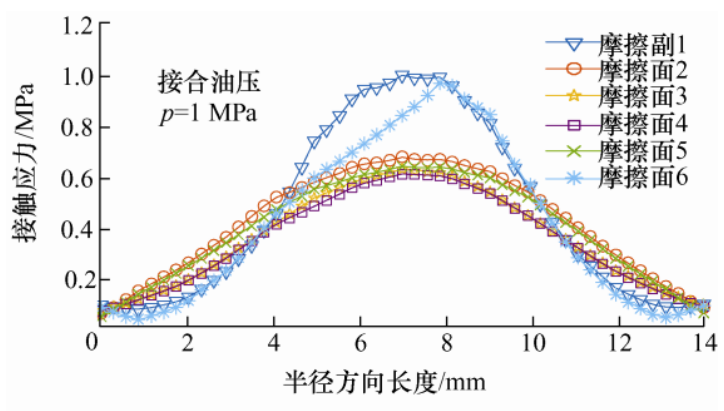

(a) 接触应力在半径方向上的分布

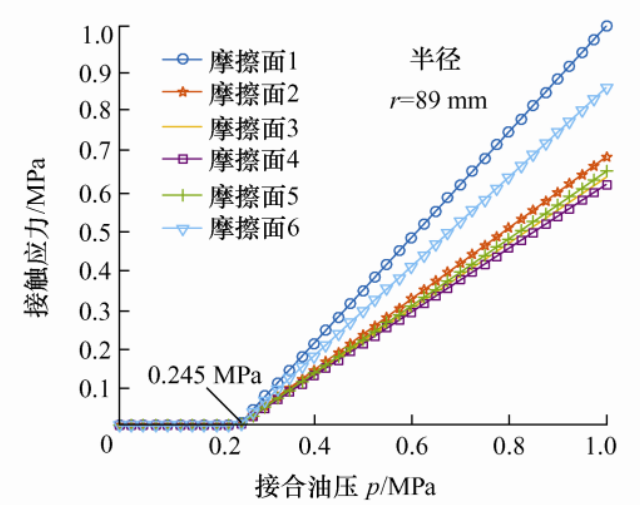

(b) 接触应力随油压变化的分布

图 4 摩擦副应力分布规律

\section{2 摩擦片沟槽内冷却流场分析}

湿式离合器摩擦片沟槽内的冷却流场受离合器 转速变化的影响, 因此摩擦片沟槽内的冷却流场在 不同转速和不同半径处的分布不同。冷却流场分布 不同会影响对流换热的效果, 为此建立了摩擦片沟 槽内冷却流场数值计算模型, 获取了摩擦片沟槽内 冷却流场的分布规律。

\section{1 摩擦片沟槽腔体化}

图 5a 为摩擦片结构, 摩擦片表面的摩擦材料与 钢片紧贴, 冷却油从摩擦片沟槽流出, 从而对钢片 进行冷却。钢片的散热能力取决于摩擦片沟槽内冷 却流场的分布。但由于本文离合器的摩擦片沟槽为 倾斜槽, 并且摩擦片不同半径处线速度不同, 因此 摩擦片沟槽内的冷却流场在不同半径处的分布不

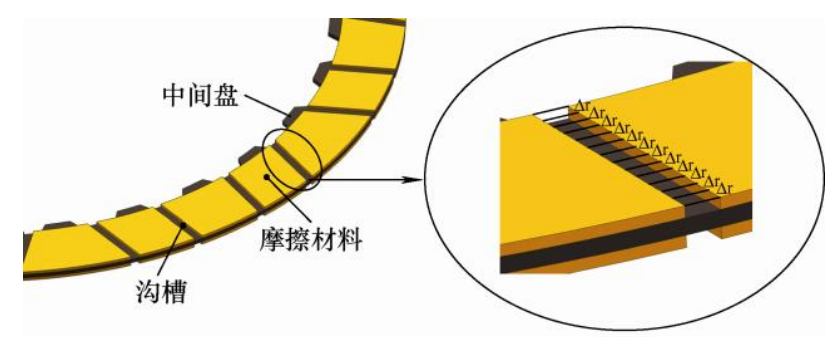

(a) 摩擦副结构

(b) 沟槽的腔体化

图 5 摩擦片沟槽结构 
同。为了能获取摩擦片沟槽内冷却流场的分布。将 沟槽等效成为厚度为 $\Delta r$ 的矩形空腔, 如图 $5 b$ 所示。 至此只要分析每个腔内的流场分布, 即可获得摩擦 片沟槽内不同半径处的流场分布。

\section{2 腔体流场模型分析}

图 6 为腔体模型, 其左、右和下边界等效为固 定面, 而上边界为等效旋转面, 等效转速为钢片与 摩擦片的转速差。

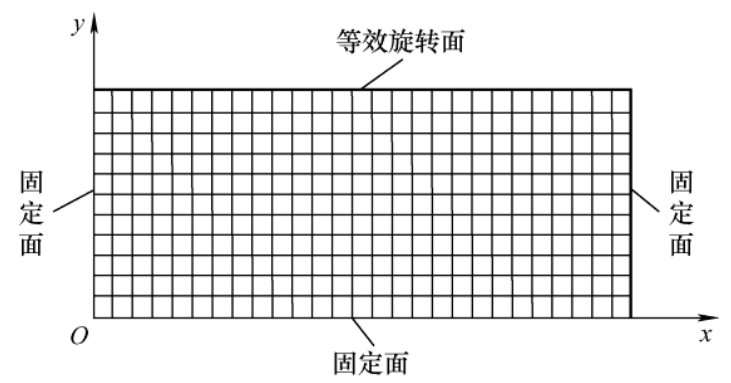

图 6 腔体模型

根据流体力学 ${ }^{[21]}$, 离合器冷却流场符合三维抛 物流, 设 $z$ 方向为主流体方向, 且忽略 $x 、 y$ 方向的 质量力。则稳态不可压缩牛顿流体 N-S 方程可以简 化为

$$
\begin{gathered}
\frac{\partial u}{\partial x}+\frac{\partial v}{\partial y}=0 \\
\rho\left(u \frac{\partial u}{\partial x}+v \frac{\partial u}{\partial y}\right)=-\frac{\partial p}{\partial x}+\mu_{o}\left(\frac{\partial^{2} u}{\partial x^{2}}+\frac{\partial^{2} u}{\partial y^{2}}\right) \\
\rho\left(u \frac{\partial v}{\partial x}+v \frac{\partial v}{\partial y}\right)=-\frac{\partial p}{\partial y}+\mu_{o}\left(\frac{\partial^{2} v}{\partial x^{2}}+\frac{\partial^{2} v}{\partial y^{2}}\right) \\
\rho\left(u \frac{\partial w}{\partial x}+v \frac{\partial w}{\partial y}\right)=\rho \omega^{2} r+\mu_{o}\left(\frac{\partial^{2} w}{\partial x^{2}}+\frac{\partial^{2} w}{\partial y^{2}}\right)
\end{gathered}
$$

式中, $u 、 v 、 w$ 为流体在直角坐标系 $x 、 y 、 z$ 方向的 速度分量； $\rho$ 为流体密度； $\mu_{o}$ 为流体动力黏度; $p$ 流 体压力; $\omega$ 为摩擦片旋转角速度; $r$ 为摩擦片半径。

如图 7 所示, 采用速度节点和压力节点分离的 交错网格和控制容积差分方法, 将式(2)离散化为

$$
\begin{gathered}
a_{P}^{u} u_{P}=a_{E}^{u} u_{E}+a_{W}^{u} u_{W}+a_{S}^{u} u_{S}+a_{N}^{u} u_{N}+\left(p_{W}-p_{P}\right) \Delta y \\
a_{E}^{u}=D_{e} A\left(\left|P_{e}\right|\right)+\max \left(-F_{e}, 0\right) \\
a_{W}^{u}=D_{w} A\left(\left|P_{w}\right|\right)+\max \left(-F_{w}, 0\right) \\
a_{N}^{u}=D_{n} A\left(\left|P_{n}\right|\right)+\max \left(-F_{n}, 0\right) \\
a_{S}^{u}=D_{s} A\left(\left|P_{s}\right|\right)+\max \left(-F_{s}, 0\right)
\end{gathered}
$$

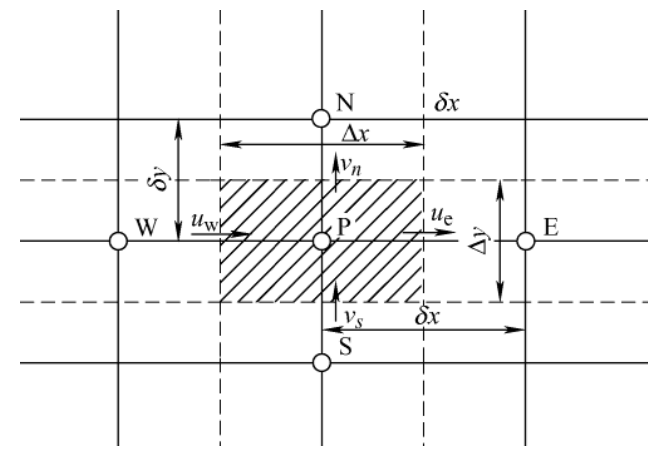

图 7 交错网格

$$
a_{P}^{u}=a_{E}^{u}+a_{W}^{u}+a_{N}^{u}+a_{S}^{u}
$$

式中, $\delta x$ 为压力节点在 $x$ 方向的间距; $\delta y$ 为压力节 点在 $y$ 方向的间距; $\Delta x$ 为速度节点在 $x$ 方向的间距; $\Delta y$ 为速度节点在 $y$ 方向的间距; $D_{e}=\mu_{o} \Delta y /(\delta x)_{e}$; $D_{w}=\mu_{o} \Delta y /(\delta x)_{w} ; \quad D_{n}=\mu_{o} \Delta x /(\delta y)_{n} ; \quad D_{s}=\mu_{o} \Delta x /(\delta y)_{s} ;$ $F_{e}=(\rho u)_{e} \Delta y ; \quad F_{w}=(\rho u)_{w} \Delta y ; \quad F_{n}=(\rho v)_{n} \Delta x ; \quad F_{s}=(\rho v)_{s} \Delta x$; $P_{e}=F_{e} / D_{e} ; \quad P_{w}=F_{w} / D_{w} ; \quad P_{n}=F_{n} / D_{n} ; \quad P_{s}=F_{s} / D_{s}$; $A(|P|)=\max \left(0,(1-|P|)^{5}\right)$ 。

采用相同的方法, 式(3)、(4)可离散化为 $a_{P}^{v} v_{P}=a_{E}^{v} v_{E}+a_{W}^{v} v_{W}+a_{S}^{v} v_{S}+a_{N}^{v} v_{N}+\left(p_{S}-p_{P}\right) \Delta x(11)$ $a_{P}^{w} w_{P}=a_{E}^{w} w_{E}+a_{W}^{w} w_{W}+a_{S}^{w} w_{S}+a_{N}^{w} w_{N}+\rho \omega^{2} r \Delta x \Delta y(12)$

将式(5)和式(11)简化, 可得速度校正公式

$$
\begin{aligned}
& u_{P}=u_{P}^{*}+\frac{\Delta y}{a_{P}^{u}}\left(p_{W}-p_{P}\right) \\
& v_{P}=v_{P}^{*}+\frac{\Delta x}{a_{P}^{v}}\left(p_{S}-p_{P}\right)
\end{aligned}
$$

式(1)在控制容积上积分, 并将式(13)和式(14) 代入可得离散化方程为

$$
\begin{gathered}
a_{P}^{p} p_{P}=a_{E}^{p} p_{E}+a_{W}^{p} p_{W}+a_{N}^{p} p_{N}+a_{S}^{p} p_{S}+b \\
b=\left(u_{w}-u_{e}\right) \rho \Delta y+\left(v_{s}-v_{n}\right) \rho \Delta x
\end{gathered}
$$

式中, $a_{E}^{p}=\rho(\Delta y)^{2} / a_{E}^{u} ; a^{p}{ }_{W}=\rho(\Delta y)^{2} / a^{u}{ }_{W} ; a^{p}{ }_{N}=\rho(\Delta x)^{2} /$ $a_{N}^{v} ; \quad a_{S}^{p}=\rho(\Delta x)^{2} / a_{S}^{v} ; \quad a_{P}^{p}=a_{E}^{p}+a^{p}{ }_{W}+a^{p}{ }_{N}+a^{p}$ 。

\section{3 三维冷却流场的求解}

三维流场求解过程如下。

(1) 给定初始压力 $p^{*}=O$ 。

(2) 将 $p^{*}$ 代入式(5)、(11), 得到 $u^{*} 、 v^{*}$ 。

(3) 求解离散化方程式(15), 得到 $p^{\prime}$ 。

(4) 由 $p=p^{*}+p^{\prime}$, 得到 $p$ 。

(5) 利用速度校正公式(13)、(14), 得到 $u 、 v$;

（6）把校正过的压力 $p$ 作为新的 $p^{*}$ ，返回到第 2 步, 重新计算直到式(16)收敛为止。 
(7) 将计算得到的 $u 、 v$ 代入式(12), 得到 $w$ 。

基于对摩擦片沟槽内三维流场的求解, 计算获 取了转速为 $2000 \mathrm{r} / \mathrm{min}$ 时, 摩擦片沟槽平均半径处 的三维流场分布。图 $8 \mathrm{a}$ 为 $u 、 v$ 在沟槽横截面内的 分布, $u 、 v$ 的矢量和在截面内形成了三个浴流, 且 三个涡流位置随着转速和半径的变化而变化, 相关 试验验证可参考文献[22-23]。图 8b 为 $w$ 在沟槽横 截面内的分布, 在沟槽中部 $w$ 数值相同, 而在沟槽 边缘处 $w$ 变化较大。

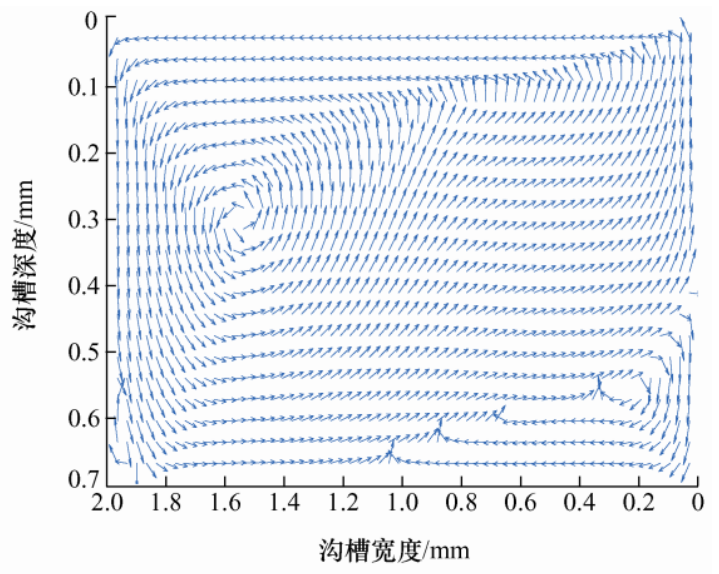

(a) $u 、 v$ 在沟槽横截面内的分布

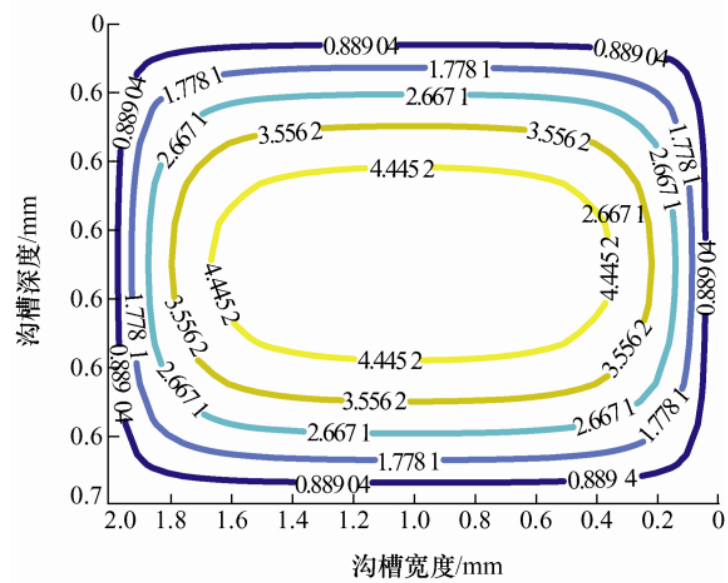

(b) $w$ 在沟槽横截面内的分布

图 8 沟槽内三维流场分布

\section{3 离合器钢片温度场分析}

基于对摩擦副接触应力场和冷却流场的分析, 建立了考虑摩擦副接触应力场和冷却流场耦合作用 的摩擦副温度场数值计算模型。

\section{1 热传导方程的建立}

湿式离合器由多对摩擦片和对偶钢片组成。图 9 为一对摩擦副传热模型, 对偶钢片两侧同时存在摩 擦热和冷却油。

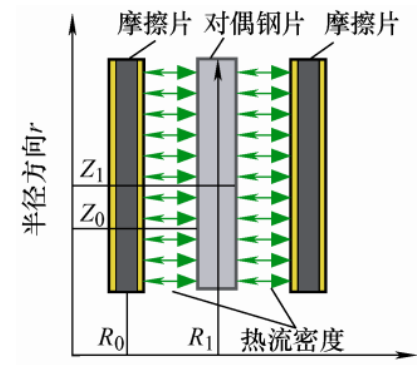

厚度方向 $z$

图 9 摩擦副传热模型

根据传热学理论 ${ }^{[24]}$, 在圆柱坐标系下的三维热 传导微分方程为

$$
\begin{gathered}
\frac{\partial}{\partial r}\left(\lambda \frac{\partial T_{i}}{\partial r}\right)+\frac{\lambda}{r}\left(\frac{\partial T_{i}}{\partial r}\right)+\frac{1}{r^{2}} \frac{\partial}{\partial \phi}\left(\lambda \frac{\partial T_{i}}{\partial \phi}\right)+ \\
\frac{\partial}{\partial z}\left(\lambda \frac{\partial T_{i}}{\partial z}\right)=\rho_{c} c \frac{\partial T_{i}}{\partial t}
\end{gathered}
$$

式中, $\lambda$ 为热导率; $\rho_{c}$ 为密度; $c$ 为比热容; $T_{i}$ 为摩 擦副的温度 $(i=1,2$ 分别为对偶钢片和摩擦片)。

假设热传导系数恒为常数, 同时摩擦副满足轴 对称热传导条件。因此可将式(17)简化为轴对称和 热导率为常数的二维热传导微分方程

$$
\frac{\partial^{2} T_{i}}{\partial r^{2}}+\frac{1}{r} \frac{\partial T_{i}}{\partial r}+\frac{\partial^{2} T_{i}}{\partial z^{2}}=\frac{1}{\alpha} \frac{\partial T_{i}}{\partial t}
$$

式中, $\alpha$ 为热扩散系数, $\alpha=\lambda /\left(\rho_{c} c\right)$ 。

对于湿式离合器在不同的接合条件下, 其边界 条件差别很大, 从而造成了离合器温度分布的不同。 以下分别讨论湿式离合器热传导方程所满足的边界 条件。

\section{2 热流密度的确定}

对于均匀的、各向同性的固体, 热量的传导满 足傅里叶定律

$$
q(r, t)=-\lambda \nabla T(r, t)
$$

由此可知只要热流密度确定, 导热条件就可确 定, 热流密度 ${ }^{[25]}$ 为

$$
q(r, t)=k \mu r p(r, t) \omega(t)
$$

式中, $\omega(t)$ 为离合器主从端转速差; $\mu$ 为摩擦因数; $p(r, t)$ 为离合器摩擦副接触应力 (第 1.3 节); $r$ 为摩擦 副半径; $k$ 表示热流分配系数 ${ }^{[26]}$, 且满足

$$
k_{d}=\frac{q_{f}}{q_{d}+q_{f}}=1-\frac{1}{1+\left(\frac{\rho_{f} c_{f} \lambda_{f}}{\rho_{d} c_{d} \lambda_{d}}\right)^{\frac{1}{2}}}
$$

式中, $f$ 表示摩擦片; $d$ 表示钢片。 


\section{3 对流换热系数的确定}

对于离合器钢片, 沟槽内冷却流场的对流换 热可近似为外掠平板强制对流换热 ${ }^{[27]}$ 。结合上述 (第 2.3 节)对摩擦片沟槽内流场的分析, 可得对流换 热系数满足

$$
\begin{gathered}
h_{s}(r, t)=0.664 \frac{\lambda}{l}(\operatorname{Re}(r, t))^{\frac{1}{2}}(\operatorname{Pr})^{\frac{1}{3}} \\
\operatorname{Re}(r, t)=\frac{u_{a}(r, t) l}{v} \\
u_{a}(r, t)=\frac{\iint \sqrt{u(r, t)^{2}+v(r, t)^{2}+w(r, t)^{2}} \mathrm{~d} x \mathrm{~d} y}{\iint \mathrm{d} x \mathrm{~d} y}
\end{gathered}
$$

式中, $l$ 为沟槽内冷却流场特征长度; $v$ 为冷却油运 动黏度; $\alpha_{o}$ 为冷却油的热扩散系数; $P r=v / \alpha_{o}$ 。

\section{4 边界条件的确定}

由于热辐射所传递的热量远小于对流换热和热 传导, 因此忽略热辐射散热对摩擦副温度场的影响, 并假设摩擦副和冷却油初始温度为 $T_{0}$ 。因此对偶钢 片温度场满足以下边界条件

$$
\begin{aligned}
& \frac{-\lambda \partial T}{\partial z}=q_{1}(r, t)-h(r, t)\left(T-T_{0}\right) \\
& z=Z_{0} \quad R_{0} \leqslant r \leqslant R_{1} \\
& \frac{\lambda \partial T}{\partial z}=q_{2}(r, t)-h(r, t)\left(T-T_{0}\right) \\
& z=Z_{1} \quad R_{0} \leqslant r \leqslant R_{1} \\
& -\lambda \frac{\partial T}{\partial r}=0 \quad r=R_{0} \quad Z_{0} \leqslant z \leqslant Z_{1} \\
& \lambda \frac{\partial T}{\partial r}=0 \quad r=R_{1} \quad Z_{0} \leqslant z \leqslant Z_{1}
\end{aligned}
$$

\section{5 离合器温度场求解}

采用交替隐式有限差分方法对热传导方程和边 界条件进行求解。求解过程如下。

(1) 将区域 $(r, z, t)$ 划分成步长为 $\Delta r 、 \Delta z 、 \Delta t$ 的 网格。

$$
\begin{gathered}
r=i \Delta r \quad i=n_{1}, n_{1}+1, n_{1}+2, \cdots, N_{1} \\
z=j \Delta z \quad j=0,1,2, \cdots, N_{2} \\
t=n \Delta t \quad n=0,1,2, \cdots, N_{3}
\end{gathered}
$$

温度 $T(r, z, t)$ 可表示为

$$
T(r, z, t)=T(i \Delta r, j \Delta z, n \Delta t)=T_{i, j}^{n}
$$

(2) 对热传导方程和边界条件进行差分。

1) 对 $r$ 进行显式差分, 对 $z$ 进行隐式差分, 差 分表达式为

$$
\begin{aligned}
& \frac{\left[\left(2-\frac{1}{i}\right) T_{i-1, j}^{n}-4 T_{i, j}^{n}+\left(2+\frac{1}{i}\right) T_{i+1, j}^{n}\right]}{2(\Delta r)^{2}}+ \\
& \frac{\left(T_{i, j-1}^{n+1}-2 T_{i, j}^{n+1}+T_{i, j+1}^{n+1}\right)}{(\Delta z)^{2}}=\frac{\left(T_{i, j}^{n+1}-T_{i, j}^{n}\right)}{\alpha \cdot \Delta t}
\end{aligned}
$$

2) 对 $r$ 进行隐式差分, 对 $z$ 进行显式差分, 差 分表达式为

$$
\begin{gathered}
\frac{\left[\left(2-\frac{1}{i}\right) T_{i-1, j}^{n+1}-4 T_{i, j}^{n+1}+\left(2+\frac{1}{i}\right) T_{i+1, j}^{n+1}\right]}{2(\Delta r)^{2}}+ \\
\frac{\left(T_{i, j-1}^{n}-2 T_{i, j}^{n}+T_{i, j+1}^{n}\right)}{(\Delta z)^{2}}=\frac{\left(T_{i, j}^{n+1}-T_{i, j}^{n}\right)}{\alpha \cdot \Delta t}
\end{gathered}
$$

3) 对边界条件进行差分

$$
\left\{\begin{array}{l}
-\lambda \frac{T_{i, 1}^{n}-T_{i, 0}^{n}}{\Delta z}=q_{1}^{n}-h^{n}\left(T_{i, 1}^{n}-T_{0}\right) \\
\lambda \frac{T_{i, N_{2}+1}^{n}-T_{i, N_{2}}^{n}}{\Delta z}=q_{2}^{n}-h^{n}\left(T_{i, N_{2}+1}^{n}-T_{0}\right), T_{i, j}^{0}=T_{0} \\
\frac{\lambda\left(T_{N_{1}+1, j}^{n}-T_{N_{1}, j}^{n}\right)}{\Delta r}=0 \frac{-\lambda\left(T_{n_{1}+1, j}^{n}-T_{n_{1}, j}^{n}\right)}{\Delta r}=0
\end{array}\right.
$$

（3）对上述差分方程进行迭代求解。

\section{6 湿式离合器温度场数值计算模型}

图 10 为湿式离合器在综合考虑摩擦副接触应 力场和冷却流场时变特性的温度场计算模型。离合 器接合过程中的转速和接合油压的变化将会导致冷 却流场和摩擦副应力场的变化, 进而影响离合器对 流换热和热流密度变化, 最终影响离合器温度场的 分布。

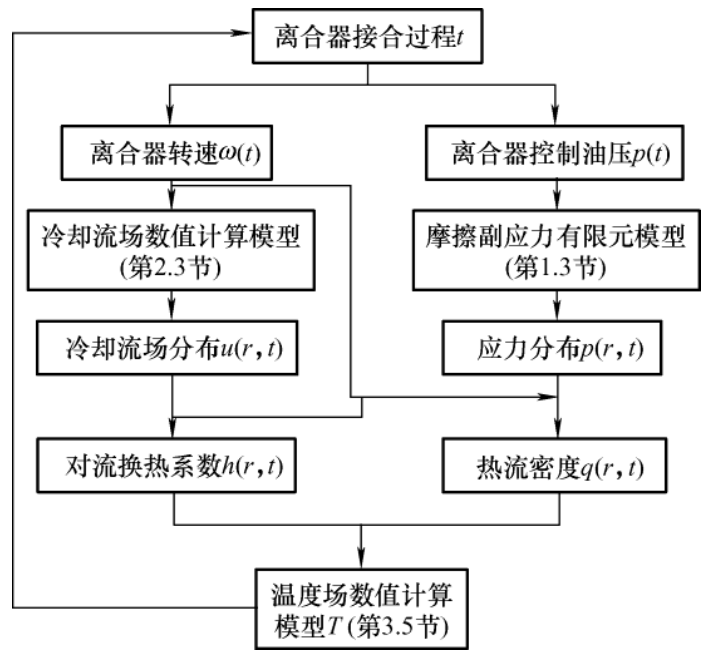

图 10 湿式离合器温度场计算模型 


\section{4 温度场计算模型的试验验证与仿真}

\section{1 湿式离合器温度场计算模型的试验验证}

为了验证第 3 节所提出的温度场计算模型的正 确性, 进行了湿式离合器温度场试验研究。

图 11 为试验系统组成。为了节约成本, 本文建 立了包含湿式离合器的变速器综合性能试验系统, 对本文提出的湿式离合器温度场计算模型进行试验 验证。

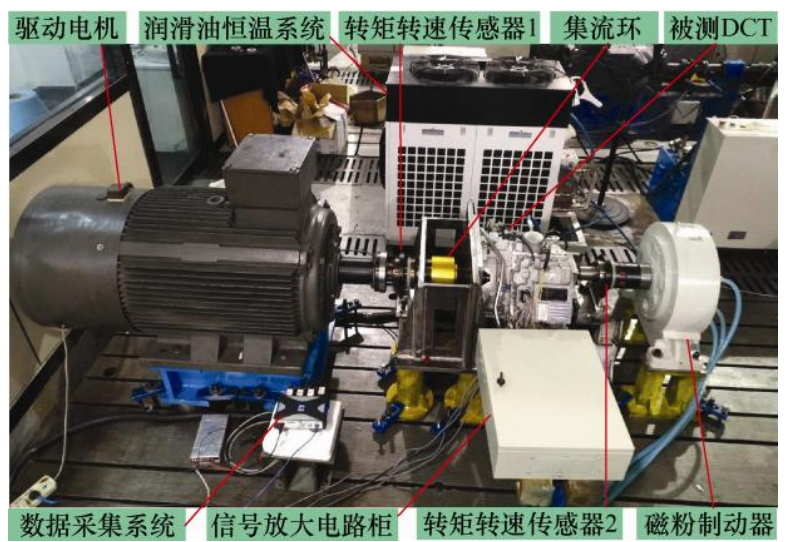

图 11 试验系统

图 12 为温度传感器的安装位置和布置形式。温 度传感器布置在离合器钢片的外径、内径以及平均 半径处。通过高温固化胶将温度传感器固定在离合 器的输入转鼓和输入轴上, 并与集流环内环相连接, 从而实现同步转动与信号的传输。

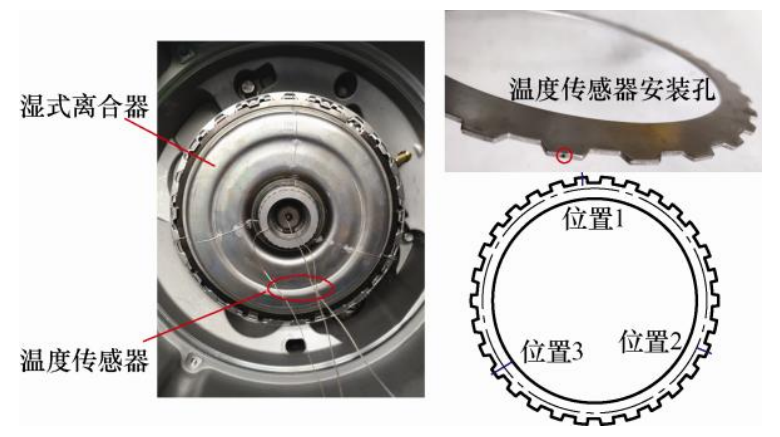

图 12 温度传感器布置

试验过程如下: 首先将驱动电机转速维持固定 值, 然后给定离合器接合油压的控制信号, 让离合 器完成一次接合, 测得离合器钢片温度、主从端转 速、主从端转矩以及接合油压信号。

图 13a 为离合器主从端转速和接合油压变化曲 线。由于油压信号存在高频波动, 不便于仿真分析, 因此对油压信号进行平滑处理。根据图 13a 的离合 器主从端转速变化、平滑的油压变化和表 1 的离合
器相关参数, 并运用第 3.6 节所提的温度场计算模 型进行仿真。

图 13b 为仿真和试验的结果对比。在离心力作 用下, 温度传感器 $(\phi 0.8 \mathrm{~mm})$ 极易扯断。试验中只获 得了离合器外径和平均半径处的温度信号。由图 $13 \mathrm{~b}$ 可知, 仿真和试验基本一致, 误差小于 $\pm 5{ }^{\circ} \mathrm{C}$ 。

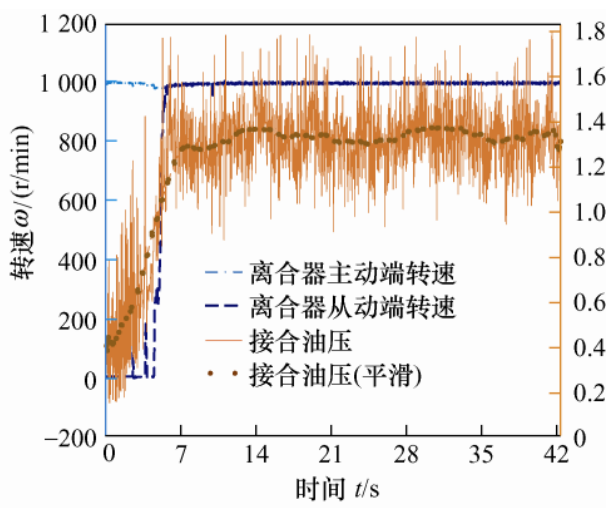

(a) 离合器转速和油压曲线

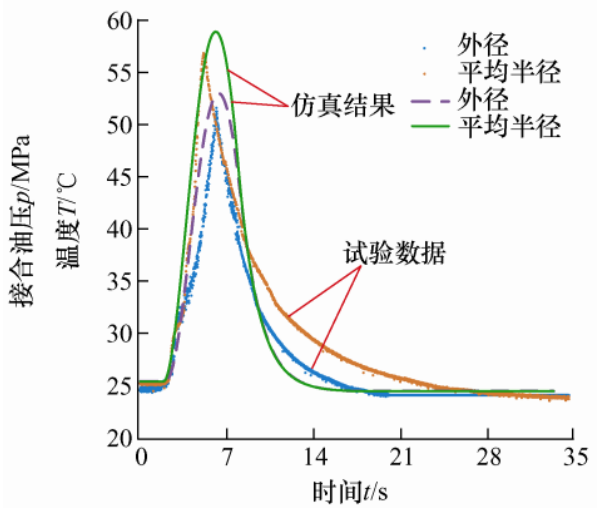

(b) 试验与仿真对比

图 13 试验验证

\section{2 湿式离合器温度场分布规律仿真分析}

对于给定的离合器, 其结构和物理参数完全确 定，如表 1 所示。

表 1 离合器相关参数

\begin{tabular}{lccc}
\hline \multirow{2}{*}{ 参数 } & \multicolumn{3}{c}{ 数 } \\
\cline { 2 - 4 } & 对偶钢片 & 摩擦片 & 润滑冷却油 \\
\hline 密度 $\rho /\left(\mathrm{kg} \cdot \mathrm{m}^{-3}\right)$ & 7800 & 1570 & 852 \\
热导率 $\lambda /\left(\mathrm{W} \cdot \mathrm{m}^{-1} \mathrm{~K}^{-1}\right)$ & 50 & 0.7 & 0.138 \\
比热容 $c /\left(\mathrm{J} \cdot \mathrm{kg}^{-1} \cdot \mathrm{K}^{-1}\right)$ & 487 & 534 & 2132 \\
\hline 离合器内径 $R_{0} / \mathrm{m}$ & \multicolumn{3}{c}{0.082} \\
离合器外径 $R_{1} / \mathrm{m}$ & \multicolumn{2}{c}{0.096} \\
润滑油动力黏度 $\mu_{0} /\left(\mathrm{m}^{2} \cdot \mathrm{s}^{-1}\right)$ & \multicolumn{2}{c}{$30 \times 10^{-6}$} \\
钢片厚度 $Z_{1} / \mathrm{m}$ & \multicolumn{2}{c}{0.002} \\
摩擦片沟槽宽 $/ \mathrm{mm}$ & \multicolumn{2}{|c}{2} \\
摩擦片沟槽深 $/ \mathrm{mm}$ & \multicolumn{2}{c}{0.7} \\
\hline
\end{tabular}

对于离合器的接合过程, 离合器接合油压和 主动端转速为控制值。在离合器负载一定的情况 
下, 离合器从动端转速由离合器接合油压确定。 现给定不同的接合油压和不同的离合器主动端

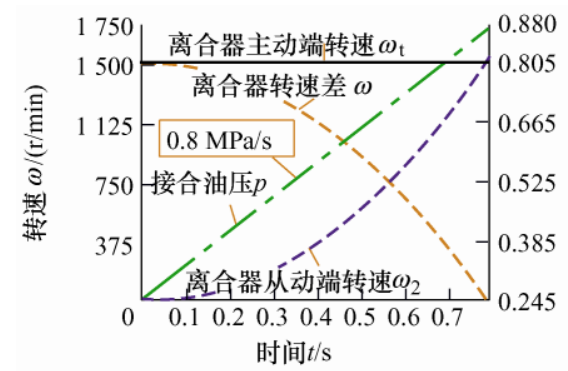

(a) $0.8 \mathrm{MPa} / \mathrm{s} 1500 \mathrm{r} / \mathrm{min}$

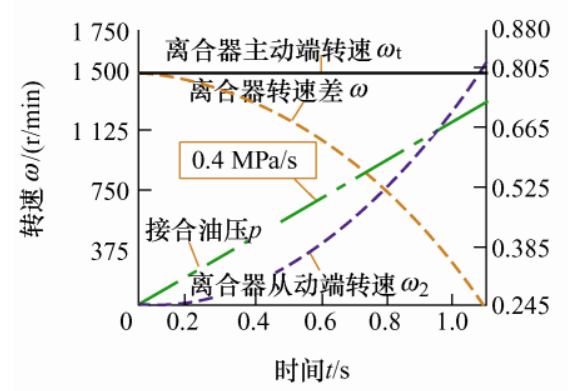

(c) $0.4 \mathrm{MPa} / \mathrm{s} 1500 \mathrm{r} / \mathrm{min}$
转速, 如图 14 所示, 对比分析离合器温度场的 变化规律。

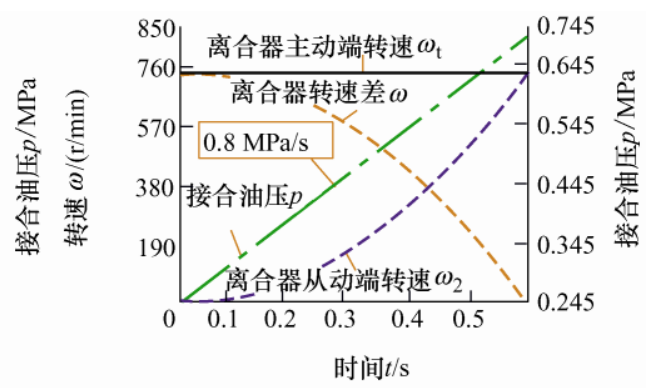

(b) $0.8 \mathrm{MPa} / \mathrm{s} 750 \mathrm{r} / \mathrm{min}$

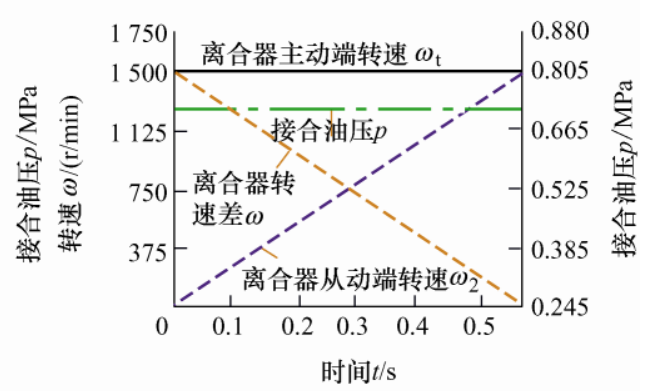

(d) $0 \mathrm{MPa} / \mathrm{s} 1500 \mathrm{r} / \mathrm{min}$

图 14 不同的接合条件

在 $\mathrm{a}$ 接合条件下, 离合器不同钢片表面温度分 布如图 15 所示。由图可知, 钢片 2 和钢片 3 的两个 表面温差均小于 $1{ }^{\circ} \mathrm{C}$, 但是钢片 1 的两个表面最高 温差为 $7{ }^{\circ} \mathrm{C}$, 钢片 4 的两个表面最高温差为 $6{ }^{\circ} \mathrm{C}$ 。 钢片 2 和钢片 3 的两个摩擦面温度分布相差很小的 原因为两个摩擦面的热量输入相差很小, 其差别是 由两个摩擦面的接触应力分布(第 1.3 节)存在微小 的差别引起的。而钢片 1 和钢片 4 只有一个摩擦面 产热, 热量由摩擦面传导到非摩擦面, 因此钢片 1 和钢片 4 的两个表面温度相差很大, 且比其他钢片 温度低。

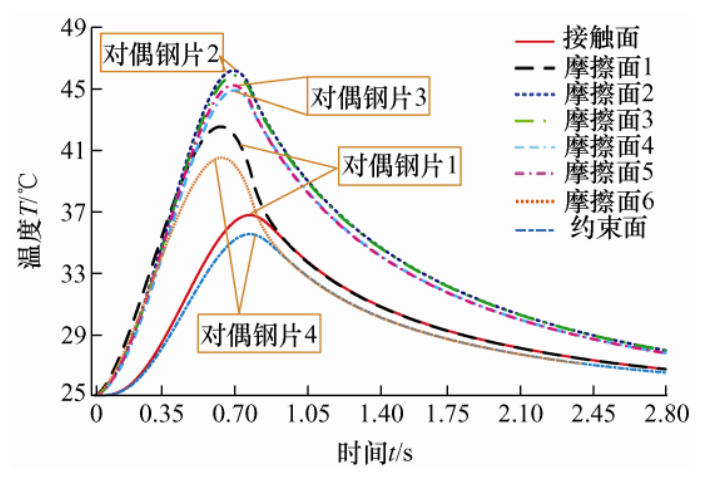

图 15 不同钢片表面温度分布

图 16 为钢片 3 的温度在厚度方向上的分布。由 图可知, 两个摩擦面的温度分布存在差值, 但差值 很小, 最大温差约为 $0.4{ }^{\circ} \mathrm{C}$ 。差值产生的原因为两 个摩擦面的接触应力分布存在微小差异引起的。同
时, 热量在厚度方向上由摩擦表面向中间传导, 因 此温度由两个表面依次向中间减小，并且由于钢片 的厚度小和导热性好, 所以温度在厚度方向上的分 布梯度很小。

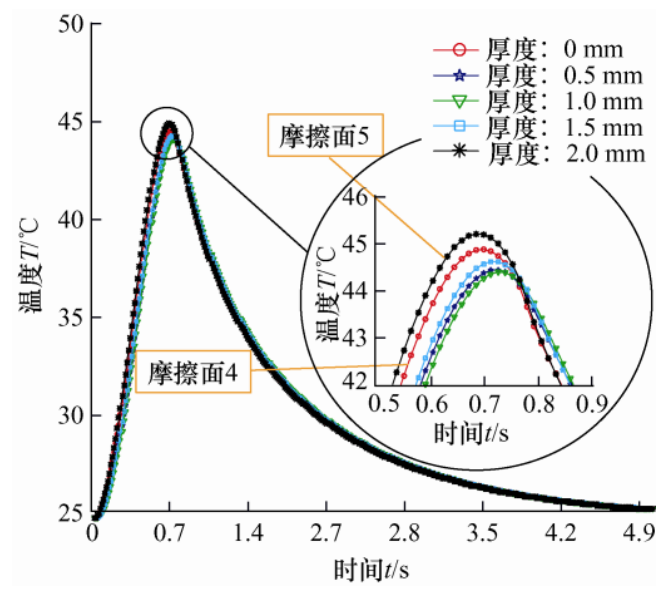

图 16 钢片温度在厚度方向上的分布

图 17 为摩擦副接触应力均匀分布和非均匀分 布(第 1.3 节)两种情况下的钢片温度场变化规律。当 摩擦副接触应力均匀分布时, 由于摩擦副的相对线 速度随着半径的增加而增加, 引起摩擦热的增加, 因此钢片的温度随着半径的增加而升高。当接触应 力非均匀分布时, 钢片温度在半径方向上的分布不 均程度明显增加, 平均半径处温度最高, 且向内、 外径方向逐渐减小, 呈抛物线分布, 其分布规律与 应力分布规律(图 4)相一致。并且, 由于摩擦副接触 
应力非均匀分布, 钢片的最高温差由 $3.41{ }^{\circ} \mathrm{C}$ (应力 均匀分布)增加到 $14.77{ }^{\circ} \mathrm{C}$, 增加了 $11.36{ }^{\circ} \mathrm{C}$ 。由此 可知, 摩擦副的接触应力分布不均会加剧离合器温 度分布不均, 温度分布不均会引起离合器摩擦副的 热弹不稳定性 ${ }^{[28]}$, 导致摩擦副的尧曲, 从而造成摩 擦副的局部热斑甚至烧蚀。而摩擦副接触应力分布 不均是由液压缸与摩擦副的接触力和固定端的约束 力为集中力造成的。因此, 对于离合器的设计应当 尽可能的保证摩擦副的接触应力在半径方向上均匀 分布, 以减小离合器温度分布的不匀, 避免离合器 的热失效问题。

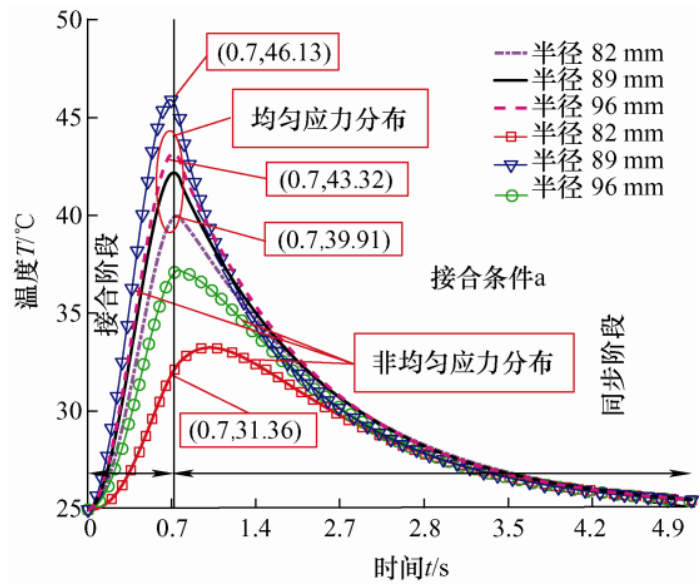

图 17 摩擦副接触应力分布对温度场的影响

图 18 对比分析了时变对流换热系数和恒定对 流换系数对钢片温度场的影响。恒定对流换热系数 采用文献[29]的数据, 而时变对流换热系数通过摩 擦片沟槽内冷却流场计算获得, 具体计算过程见第 2.3 节。在离合器接合阶段, 时变对流换热和恒定对 流换热两种情况下的钢片温度分布完全一致。但在 离合器同步阶段, 由于仅存在对流换热, 并且随着 摩擦片转速的升高, 对流换热系数相应的要增加, 因此时变对流换热和恒定对流换热两种情况下的钢 片温度分布将产生差值, 差值由离合器同步前

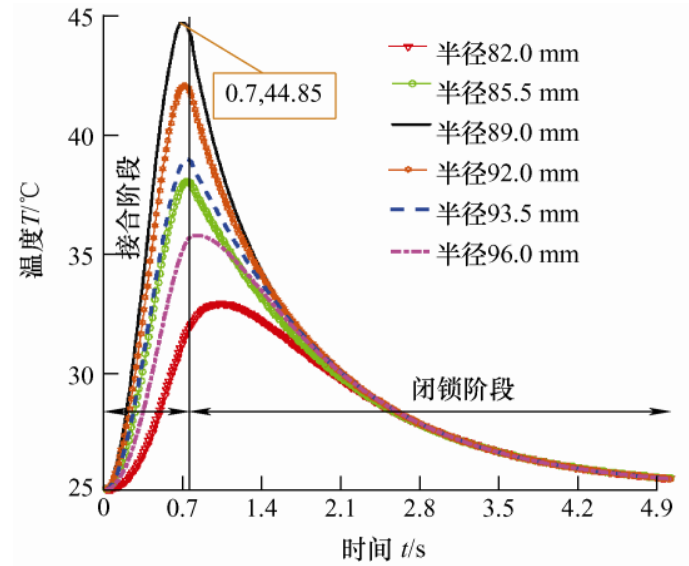

(a) $0.8 \mathrm{MPa} / \mathrm{s} 1500 \mathrm{r} / \mathrm{min}$
的 $0.66{ }^{\circ} \mathrm{C}$ 增加到离合器同步阶段的 $4.5{ }^{\circ} \mathrm{C}$ 。由此可 以发现, 冷却流场分布主要对离合器同步阶段的温 度分布规律影响较大。

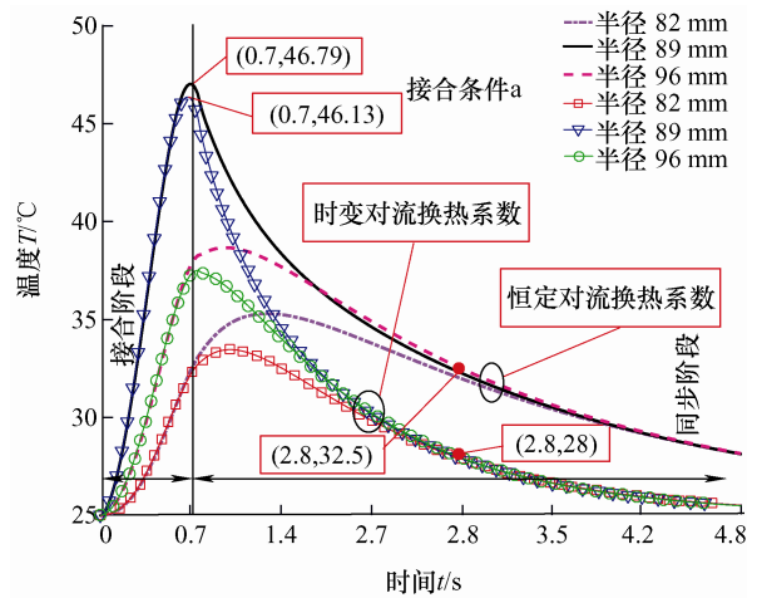

图 18 冷却流场分布对温度场的影响

图 19 为 $a 、 b 、 c 、 d$ 四种接合条件下离合器温 度场分布规律。由图 19a 和图 19b 对比分析可知, 离合器主动端转速从 $750 \mathrm{r} / \mathrm{min}$ 增加到 $1500 \mathrm{r} / \mathrm{min}$ 时, 离合器对偶钢片的最高温度从 $30.79{ }^{\circ} \mathrm{C}$ 增加到 $44.85{ }^{\circ} \mathrm{C}$, 增加了 $14.06{ }^{\circ} \mathrm{C}$ 。由图 $19 \mathrm{a}$ 和图 $19 \mathrm{c}$ 对 比分析可知, 离合器接合油压变化率从 $0.4 \mathrm{MPa} / \mathrm{s}$ 增加到 $0.8 \mathrm{MPa} / \mathrm{s}$ 时, 离合器最高温度从 $43.35{ }^{\circ} \mathrm{C}$ 增 加到 $44.85{ }^{\circ} \mathrm{C}$, 增加了 $1.5{ }^{\circ} \mathrm{C}$, 但是离合器的接合 时间缩短了。由图 19c 和图 19d 对比分析可知, 在 离合器接合油压以斜率 $0.4 \mathrm{MPa} / \mathrm{s}$ 线性增加和维持 恒定两种情况下, 离合器最高温度从 $43.35{ }^{\circ} \mathrm{C}$ 增加 到 $43.88{ }^{\circ} \mathrm{C}$, 增加了 $0.53{ }^{\circ} \mathrm{C}$ 。因此, 对于离合器的 接合过程, 离合器转速差的变化对离合器温度场分 布的影响相比离合器接合油压更为明显。对于离合 器的接合过程, 在满足离合器工作需求的情况下, 可以通过合理控制离合器主动端转速, 减小转速差, 进而控制离合器最高温升, 以避免离合器的烧蚀。

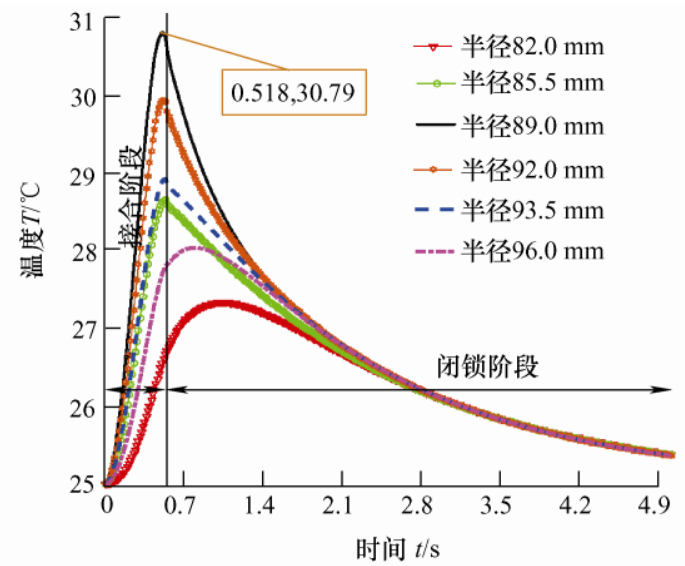

(b) $0.8 \mathrm{MPa} / \mathrm{s} 750 \mathrm{r} / \mathrm{min}$ 


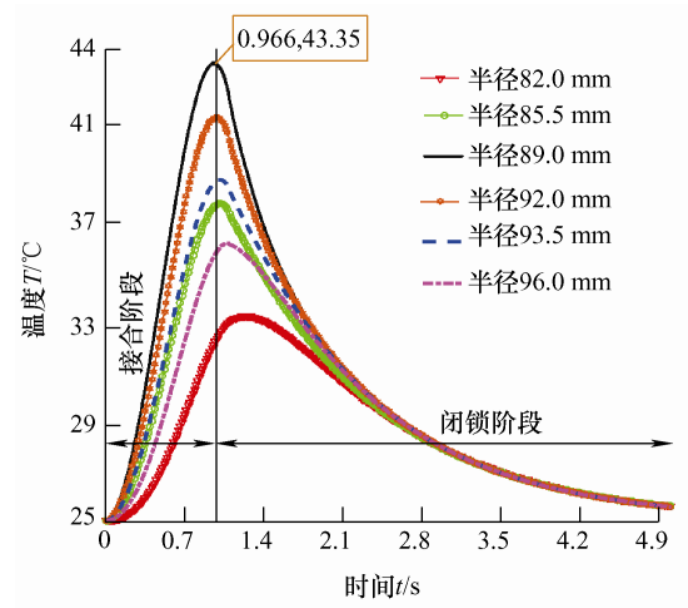

(c) $0.4 \mathrm{MPa} / \mathrm{s} 1500 \mathrm{r} / \mathrm{min}$

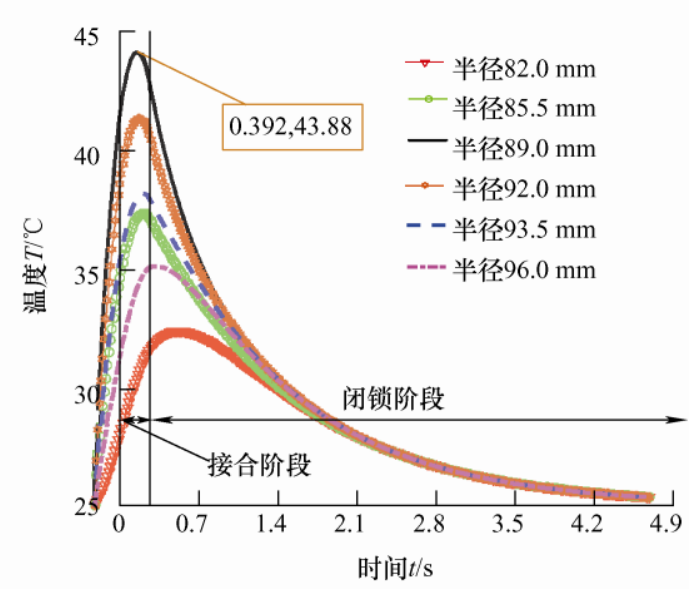

(d) $0 \mathrm{MPa} / \mathrm{s} 1500 \mathrm{r} / \mathrm{min}$

图 19 不同接合条件下离合器温度场分布规律

\section{5 结论}

(1) 本文提出了一种综合考虑摩擦副接触应力 场和冷却流场时变特性的离合器温度场数值计算模 型。应用该模型可分析摩擦副接触应力场和冷却流 场对离合器温度场的影响规律。

(2) 对于离合器接合过程, 离合器温度场在摩 擦副半径方向上的分布规律与摩擦副应力分布规 律相一致。而冷却流场分布主要对离合器同步阶 段的温度分布规律影响较大, 决定了离合器同步 阶段温度下降的快慢。对于离合器的接合过程, 离合器转速差的变化对离合器的最高温度影响 较大。

\section{参 考 文 献}

[1] 赵治国, 顾佳鼎, 何露. 干式双离合器自动变速器换档 过程离合器传递转矩估计 [J]. 机械工程学报, 2017, 53(14): 77-87.

ZHAO Zhiguo, GU Jiading, HE Lu. Estimation of torques transited by twin-clutch during shifting process for dry dual clutch transmission[J]. Journal of Mechanical Engineering, 2017, 53(14): 77-87.

[2] JEN T C, NEMECEK D J. Thermal analysis of a wet-disk clutch subjected to a constant energy engagement[J]. International Journal of Heat \& Mass Transfer, 2008, 51(7-8): 1757-1769.

[3] ZHU Zhencai, PENG Yuxing, SHI Zhiyuan. Three-dimensional transient temperature field of brake shoe during hoist's emergency braking[J]. Applied Thermal Engineering, 2009, 29(5-6): 932-937.

[4] PACEY D A, TURNQUIST R O. Modeling heat transfer in a wet clutch[R]. SAE，9016539，1990.

[5] PISATURO M , SENATORE A. Simulation of engagement control in automotive dry-clutch and temperature field analysis through finite element model[J]. Applied Thermal Engineering, 2015, 93: 958-966.

[6] YANG Yubo, LAM R C. Theoretical and experimental studies on the interface phenomena during the engagement of automatic transmission clutch[J]. Tribology Letters, 1998, 5(1): 57-67.

[7] TATARA R A, PARVIZ P. Multiple engagement wet clutch heat transfer model[J]. Numerical Heat Transfer, Part A: Applications, 2002, 42(3): 215-231.

[8] LI Wenbin, HUANG Jianfeng, FEI Jie, et al. Simulation and application of temperature field of carbon fabric wet clutch during engagement based on finite element analysis[J]. International Communications in Heat and Mass Transfer, 2016, 71: 180-187.

[9] ADAMOWICZ A, GRZES P. Analysis of disc brake temperature distribution during single braking under non-axisymmetric load[J]. Applied Thermal Engineering, 2011, 31(6): 1003-1012.

[10] YU Liang, MA Biao, LI Heyan, et al. Numerical and experimental studies of a wet multidisc clutch on temperature and stress fields excited by the concentrated load[J]. Tribology Transactions, 2018, 62(1): 8-21.

[11] ZAGRODZKI P. Thermoelastic instability in friction clutches and brakes - Transient modal analysis revealing mechanisms of excitation of unstable modes[J]. International Journal of Solids and Structures, 2009, 46(11-12): 2463-2476. 
[12] ZAGRODZKI P, TRUNCONE S A. Generation of hot spots in a wet multidisk clutch during short-term engagement[J]. Wear, 2003, 254(5): 474-491.

[13] ZAGRODZKI P. Numerical analysis of temperature fields and thermal stresses in the friction discs of a multidisc wet clutch[J]. Wear, 1985, 101(3): 255-271.

[14] ZAGRODZKI P. Influence of design and material factors on thermal stresses in multiple disc wet clutches and brakes[R]. SAE，911833， 1991.

[15] ZAGRODZKI P, FARRIS T D. Analysis of temperatures and stresses in wet friction disks involving thermally induced changes of contact pressure[R]. SAE, 982035, 1998.

[16] ZHAO Shuangmei, HILMAS G E, DHARANI L R. Behavior of a composite multidisk clutch subjected to mechanical and frictionally excited thermal load[J]. Wear, 2008, 264(11): 1059-1068.

[17] CUI Jianzhong, WANG Cuntang, XIE Fangwei, et al. Numerical investigation on transient thermal behavior of multidisk friction pairs in hydro-viscous drive[J]. Applied Thermal Engineering, 2014, 67(1-2): 409-422.

[18] YANG Yubo, LAM R C, CHEN Y F, et al. Modeling of heat transfer and fluid hydrodynamics for a multidisc wet clutch[R]. SAE, 950898, 1995.

[19] LAI Y G. Simulation of heat-transfer characteristics of wet clutch engagement processes[J]. Numerical Heat Transfer, Part A: Applications, 1998, 33(6): 583-597.

[20] MAHMUD S F, PAHLOVY S A, OGAWA M. Simulation to estimate the output torque characteristics and temperature rise of a transmission wet clutch during the engagement process[R]. SAE, 2018-01-0402， 2018.

[21] PATANKAR S V. Numerical heat transfer and fluid flow[M]. New York: McGraw-Hill, 1980.
[22] KREIZER M, RATNER D, LIBERZON A. Real-time image processing for particle tracking velocimetry $[\mathrm{J}]$. Experiments in Fluids, 2010， 48(1): 105-110.

[23] LIBERZON A, FELDMAN Y, GELFGAT A Y. Experimental observation of the steady - oscillatory transition in a cubic lid-driven cavity[J]. Physics of Fluids, 2011, 23(8): 1-7.

[24] OZISIK M N. Heat conduction[M]. New Jersey : John-Wiley, 1980

[25] LING F F, GOODMAN L E. Surface mechanics[J]. Journal of Applied Mechanics, 1974, 41(4): 1149-1151.

[26] GRZES P, MECHANIKI K，STOSOWANEJ I. Partition of heat in 2D finite element model of a disc brake[J]. Acta Mechanica Et Automatica， 2011， 5: 35-41.

[27] MILLS A F. Heat transfer[M]. New Jersey: Prentice Hall, 1999.

[28] 马彪, 赵家昕, 陈漫, 等. 摩擦材料特性对离合器热弹 性不稳定性的影响 [J]. 机械工程学报, 2014, 50(8): 111-118.

MA Biao, ZHAO Jiaxin, CHEN Man, et al. Effect of friction material properties on thermoelastic instability of clutches[J]. Journal of Mechanical Engineering, 2014, 50(8): 111-118

[29] MARKLUND P， SAHLIN F， LARSSON R. Modelling and simulation of thermal effects in wet clutches operating under boundary lubrication conditions[J]. Proceedings of the Institution of Mechanical Engineers, Part J: Journal of Engineering Tribology, 2009, 223(8): 1129-1141.

作者简介: 吴邦治, 男, 1993 年出生, 博士研究生。主要研究方向为车 辆动力传动与控制。

E-mail: bangzhiwu@cqu.edu.cn

秦大同(通信作者), 男, 1956 年出生, 博士, 教授, 博士研究生导师。 主要研究方向为车辆动力传动与控制。

E-mail: dtqin@cqu.edu.cn 